\title{
REPRESENTING MEASURES FOR POINTS IN A UNIFORM ALGEBRA
}

\author{
BY ERRETT BISHOP \\ Communicated by Walter Rudin, September 9, 1963
}

Recent years have seen much effort put into attempts to develop an "abstract" function theory. In the complex domain, this has led to the study of the structure of uniform algebras. A uniform algebra $\mathfrak{A}$ is a family of continuous complex-valued functions on a compact Hausdorff space $X$, which contains the function 1, which is closed with respect to the algebraic operations of addition and multiplication by complex scalars, which is topologically closed in the uniform norm, and which distinguishes points of $X$. Succinctly, $\mathfrak{A}$ is a closed separating unitary subalgebra of the Banach algebra $C(X)$ of all continuous complex-valued functions on $X$. Standard examples are obtained by taking $X$ to be a compact subset of complex Euclidean space $C^{n}$ and $\mathfrak{A}$ to be the closed subalgebra of $C(X)$ generated by the constants and the coordinate functions on $C^{n}$.

Some of the most suggestive ideas in the theory of uniform algebras come from a paper of Gleason [1]. He calls elements $x$ and $y$ in $X$ equivalent if

$$
d(x, y)=\sup \{|f(x)-f(y)|: f \in \mathfrak{A},\|f\| \leqq 1\}<2 .
$$

This is an equivalence relation on $X$. The equivalence classes are called parts or Gleason parts. The parts have various other characterizations, obtainable from the elementary conformal geometry of the $\operatorname{disc} D=\{z:|z| \leqq 1\}$. We shall need to know, in particular, that if there exists a sequence $\left\{h_{n}\right\}$ of elements of $\mathfrak{A},\left\|h_{n}\right\| \leqq 1,\left|h_{n}(x)\right| \rightarrow 1$ as $n \rightarrow \infty$, then $\left|h_{n}(y)\right| \rightarrow 1$ as $n \rightarrow \infty$ if $x$ and $y$ are in the same part.

Gleason showed, in a special case, that parts can be given a certain characterization in terms of representing measures. A representing measure $\mu_{x}$ for a point $x$ in $X$ is a non-negative Baire measure on $X$ such that $\int f d \mu_{x}=f(x)$ for all $f$ in $\mathfrak{A}$. The following theorem generalizes Gleason's result to the general situation.

TheOREM 1. If $x$ and $y$ are in the same part, there exists $c>0$ and representing measures $\mu_{x}$ for $x$ and $\mu_{y}$ for $y$ such that $c \mu_{x} \leqq \mu_{y}$ and $c \mu_{y} \leqq \mu_{x}$.

Proof. Let $C_{r}(X)$ be the Banach space of all continuous realvalued functions on $X$. Let $R$ be the set of all $f$ in $C_{r}(X)$ such that $f+i g \in \mathfrak{A}$ for some $g$ in $C_{r}(X)$. Let $c$ be a constant, $0<c<1$, such that 
there exists $f$ in $R$ with $f \geqq 0, f(y)=1$, and $f(x)<c$. Then with $g$ as above we have

$$
h=e^{-(f+i g)} \in \mathfrak{A},
$$

$\|h\| \leqq 1,|h(y)|=e^{-1},|h(x)| \geqq e^{-c}$. Now if $c$ can be chosen arbitrarily near to 0 we see from the above that $x$ and $y$ would not be in the same part. Thus there exists $c<1$ such that for all $f$ in $R, f \geqq 0$, we have $f(x) \geqq c f(y)$. For reasons of symmetry we may choose the constant $c$ so that in addition $f(y) \geqq c f(x), f$ in $R, f \geqq 0$. By a standard theorem, it follows that there exists a positive measure $\alpha$ on $X$ such that $f(x)-c f(y)=\int f d \alpha$ for all $f$ in $R$, and thus for all $f$ in $\mathfrak{A}$. Similarly, there exists a positive measure $\beta$ on $X$ such that $\int f d \beta=f(y)-c f(x)$ for all $f$ in $\mathfrak{A}$. Thus

$$
f(y)=c f(x)+\int f d \beta=c^{2} f(y)+c \int f d \alpha+\int f d \beta,
$$

so

$$
f(y)=\left(1-c^{2}\right)^{-1}\left[c \int f d \alpha+\int f d \beta\right] .
$$

Similarly,

$$
f(x)=\left(1-c^{2}\right)^{-1}\left[c \int f d \beta+\int f d \alpha\right] .
$$

Thus the theorem holds with

$$
\begin{aligned}
& \mu_{x}=\left(1-c^{2}\right)^{-1}[c d \beta+d \alpha], \\
& \mu_{y}=\left(1-c^{2}\right)^{-1}[c d \alpha+d \beta] .
\end{aligned}
$$

COROLLARY. If $\mu_{x}^{\prime}$ is any representing measure for $x$, there exists a representing measure $\mu_{y}^{\prime}$ for $y$ with $c \mu_{x}^{\prime} \leqq \mu_{y}^{\prime}$.

PRoor. Take $\mu_{x}$ and $\mu_{y}$ as above, and write $\mu_{y}^{\prime}=\left(\mu_{y}-c \mu_{x}\right)+c \mu_{x}^{\prime}$.

We remark that a lower bound for $c$ could be explicitly computed in terms of $d(x, y)$.

\section{REFERENCES}

1. A. Gleason, Function algebras, Seminars on analytic functions, Inst. for Advanced Study, Princeton, N. J., 1957, vol. 2.

Untversity of California, Berkeley 